\title{
Compared performances of four algorithms used for modulation thermography
}

\author{
by J.-C. Krapez
}

ONERA, DMSE, BP 72, F-92322 CHÂTILLON-Cedex, France, e-mail: krapez@onera.fr

\begin{abstract}
A theoretical analysis is presented about the performances of a series of four different algorithms for modulation thermography: standard lock-in method, 4-bucket method, variance method and leastsquares method. The precision on the amplitude and on the phase lag is evaluated versus the number of integrated images; depending on the input noise level, on the actual signal amplitude and on the quantisation level.
\end{abstract}

\section{Introduction}

In the eighties-nineties some attempts were made to adapt conventional lock-in procedures to thermography so that modulated thermal fields could be controlled, i.e. the socalled thermal waves. Pioneering work was performed at Reims and Marseille Universities $[1,2]$. Real-time lock-in thermography was later reported [3-5]. Other works later pinpointed some peculiarities originating from the sampling nature of signal collection: the existence of forbidden frequencies and of "alliasing collision frequencies" [6,7]. Two other methods emerged a few years ago: the 4-bucket lock-in method [8] and a statistical method [9,10]. The later one is not strictly a lock-in technique since there is no reference signal and consequently no phase-sensitive detection. For this reason only the signal amplitude map is retrieved, not the phase map. Nevertheless we decided to add this method to our performance analysis.

It was not until mid-nineties before a series of commercial instruments were proposed to perform multiplex lock-in thermography. One can mention the Agema Thermovision 800 monodetector cameras [11] (4-bucket method), the Cedip FPA cameras [12] and the DeltaTherm ${ }^{\mathrm{TM}} 1000$ FPA camera from Stress Photonics.

Our purpose was to analyse the performance of some algorithms suggested for modulation thermography. Basically the lock-in thermography system collects a given number of images $\mathrm{N}$ from which an amplitude and a phase lag are calculated for each pixel. It is expected that the standard deviation for amplitude and phase decreases as $N^{-1 / 2}$. Our objective was to evaluate in more detail the noise reduction for the following algorithms:

1- standard lock-in method (SLIM) $[3-5,13]$ 3-variance method (VM) $[9,10]$

2- 4-bucket method (4BM) $[8,11,13-15] \quad$ 4- least squares method (LSM) [2, 13]

Preliminary comparative results for SLIM, 4BM and LSM were already published [13]. They showed in particular that for SLIM it is important to first subtract the mean level from the signal (this procedure was since then implemented in Cedip lock-in systems).

\section{Compared algorithms}

\subsection{Standard lock-in method (SLIM)}

It relies on the multiplication of the signal $s(t)$ by the in-phase and in-quadrature reference signals $p_{f}(t)=\sin \left(2 \pi f t+\varphi_{r}\right)$ and $q_{f}(t)=\cos \left(2 \pi f t+\varphi_{r}\right)$ and then on separate summation of these results in $S P_{f}$ and $S Q_{j}$.

$$
S P_{f}=\sum_{i=1}^{N} s\left(t_{i}\right) p_{f}\left(t_{i}\right) \quad S Q_{f}=\sum_{i=1}^{N} s\left(t_{i}\right) q_{f}\left(t_{i}\right)
$$


Amplitude $A$ and phase $\varphi$ of the component of $s(t)$ which is at frequency $f$ are obtained from:

$$
\operatorname{tg}\left(\varphi-\varphi_{r}\right)=\frac{S Q_{f}}{S P_{f}} \quad A=\frac{2}{N} \sqrt{S P_{f}^{2}+S Q_{f}^{2}}
$$

\subsection{The 4-bucket method (4BM)}

The signal is sampled at four times the reference frequency and the results are respectively averaged in $S_{l} \ldots S_{+}$. Amplitude and phase of the signal are obtained by:

$$
\operatorname{tg}(\varphi)=\frac{S_{1}-S_{3}}{S_{2}-S_{4}} \quad \cdot \quad A=\frac{1}{2} \sqrt{\left(S_{1}-S_{3}\right)^{2}+\left(S_{2}-S_{4}\right)^{2}}
$$

Basically the driving phenomenon (sample heating, mechanical constraints, ...) is monitored by the camera itself at $f_{c} / 4$ where $f_{c}$ is the camera scanning frequency [8]. Faster phenomena modulated at $f_{c}(m+1 / 4)$ or at $f_{c}(m+3 / 4)$ can be analysed through undersampling [13]. On the opposite, for very low phenomena, integration of all the images contained in one quarter [14] or one half [13] of camera period is of course advisable (expression for the amplitude in (3) should be accordingly modified). In the following we will only consider the case of a modulation at $f_{c}(m+1 / 4)$ with $m \geq 0$.

\subsection{Variance method (VM)}

This method requires no reference signal, eliminating by this way any synchronisation constraint. It however only provides information on the amplitude. Assuming that the noise of the instrument and the useful signal are uncorrelated, and that the thermal modulation is sinusoïdal, its amplitude is given by $A=\sqrt{2\left(V_{s}-V_{b}\right)}$ where $V_{s}$ is the experimental signal variance and $\mathrm{V}_{\mathrm{b}}$ the noise variance. As only estimates can be obtained for both variances, we used the modified expression: $A=\sqrt{2 M a x}\left[0, V_{s}-V_{b}\right]$.

\subsection{Least squares method (LSM)}

We developed this method for processing the IR films obtained with our monodetector or FPA cameras. No synchronisation is required between the camera and the driving phenomenon. Best results are obtained with the FPA camera as in that case we simultaneously record the reference signal for precise timing.

We calculate the amplitude and the phase of a sinusoidal signal perturbed by noise by minimising the square difference between the experimental signal and the theoretical one. In addition to $S P_{f}$ and $S Q_{f}$ we need to accumulate the phase and quadrature reference functions in "buffers" $P_{f}$ and $Q_{f}$, the reference functions with double pulsation in $P_{2 f}$ and $Q_{2 f}$, and the signal itself in $S$ (e.g.: $P_{f}=\sum_{i=1}^{N} p_{f}\left(t_{i}\right)$ ). Their presence is due to the fact that the number of images does not necessary correspond to an integral number of periods.

Phase and amplitude are retrieved through:

$$
\operatorname{tg}\left(\varphi-\varphi_{r}\right)=\operatorname{tg} \Delta \varphi=\frac{\overline{S Q_{f}}\left(\bar{N}-\overline{Q_{2 f}}\right)-\overline{S P_{f} P_{2 f}}}{\overline{S P_{f}}\left(\bar{N}+\overline{Q_{2 f}}\right)-\overline{S Q_{f} P_{2 f}}}
$$


with:

$$
A=2 \frac{\overline{S P_{f}} \cos \Delta \varphi+\overline{S Q_{f}} \sin \Delta \varphi}{\bar{N}-\overline{Q_{2 f}} \cos 2 \Delta \varphi+\bar{P}_{2 f} \sin 2 \Delta \varphi}
$$

$$
\begin{array}{ll}
\overline{S P_{f}}=S P_{f}-P_{f} S / N & \overline{P_{2 f}}=P_{2 f}-2 P_{f} Q_{f} / N \\
S Q_{f}=S Q_{f}-Q_{f} S / N & \overline{Q_{2 f}}=Q_{2 f}+\left(P_{f}^{2}-Q_{f}{ }^{2}\right) / N \\
\bar{N}=N-\left(P_{f}{ }^{2}+Q_{f}{ }^{2}\right) / N &
\end{array}
$$

One important point is that SLIM, VM, and LSM present the same "forbidden" frequencies which are the multiples of the Nyquist frequency $f_{c} / 2$. By introducing $\langle f\rangle$ the fractional part of the division of $f$ by $f_{c},\langle f>$ should not be too close to $0,0.5$ or 1 .

\section{Monte-Carlo simulations}

The performances of these algorithms were evaluated through Monte-Carlo simulations. The discrepancy between the actual amplitude and phase lag of the pure signal and their counterpart obtained by lock-in were calculated on a statistical basis: a large amount of virtual experiments (from 500 to 12500) were repeated by randomly setting the absolute phase values of the signal and of the reference. For SLIM and LSM the frequency was also randomly selected (this selection was performed on the $\langle f\rangle$ value by choosing it 0.05 away from the "forbidden" values $0,0.5$, and 1). Gaussian noise was added to the pseudoexperimental "analogic" signal. Then a A/D conversion was simulated. The different algorithms were applied from this stage on.

Different values were considered for the true signal amplitude and for the noise standard deviation before the A/D conversion. These values were selected with reference to the interval between two levels of quantisation (in digit numbers). This allowed us to analyse the lock-in efficiency in the case of moderate and low S/N level, in the presence of "crude" or "fine" A/D conversion.

The standard deviation of the phase lag versus the number of images $\mathrm{N}$ is shown in fig.1. The standard deviation of the amplitude (output noise) was ratioed with the input noise level to obtain the noise attenuation factor of the considered algorithm (fig. 2).

\section{Results and discussion}

There are marked differences between the four algorithms. The efficiency improvement with the increase of the number of images depends on the chosen method. Furthermore, the fact that the input noise is larger or lower than one digit level can have high impact on the results. One can notice the following behaviours.

For the standard lock-in method (SLIM) the $N^{-1 / 2}$ trend of the noise attenuation factor is only observed when the signal amplitude is low (less than 10 digits when the input noise level is of 3 digits). The filtering capability of the lock-in approach becomes worse as the amplitude rises. This is particularly effective for low input noise $(0.3$ digit level). The efficiency loss is also observed for the phase: in the case of high signal amplitude the phase standard deviation is higher than with other methods. These shortcomings are due to the fact that the expressions (3) and (4) are only approximate.

The noise attenuation factor shows a lower limit for $N>1000$ in the case of low amplitude and low input noise. This behaviour is anyhow observed for all methods. It is a consequence of the quantisation procedure.

The 4-bucket method (4BM) presents good results in the case of 3 digits input noise. For 0.3 digit input noise a lower limit for the noise attenuation factor is now observed for any signal amplitude value. A similar levelling-off is also observed for the decrease of the phase standard deviation. 
The Variance method (VM) presents several shortcomings. The noise attenuation factor in the case of high amplitude signal reaches standard values only for high $N$ values. On the opposite, for low amplitude signals, the noise attenuation trend is never as steep as $N^{-1 / 2}$.

The Least squares method (LSM) presents nearly ideal results: the noise attenuation factor and the phase standard deviation are always like $N^{-1 / 2}$ if one excludes a saturation of the first parameter for low signal amplitude and low input noise.

\section{Conclusion}

Theoretical simulations revealed that the algorithms for modulation thermography one can find in the literature do not show the same efficiency regarding amplitude and phase lag calculation. In this paper we only considered the case of a sinusoïdal IR signal. The influence of higher harmonics will be presented in a forthcoming paper. The four methods that we considered can be sorted in increasing order of efficiency: the variance method (VM), the standard lock-in method (SLIM), the 4-bucket method (4M) and the least-squares method (LSM). VM provides only amplitude data and $4 \mathrm{M}$ presents severe limitations regarding the "allowed" frequencies. One can thus conclude that the most precise and most versatile method is the least-squares method.

\section{REFERENCES}

[1] BEAUDOIN (J.-L.), MERIENNE (E.), DANJOUX (R.) and EGEE (M.) - Proc. SPIE, vol. 590, Infrared Technology and Applications, 1985, p. 285-292.

[2] BOUC (R.) and NAYROLES (B.), DE MÉC (J.) - Théor. et Appl., vol. 4(1), 1985, p. 27-58.

[3] KUO (P. K.), FENG (Z. J.), AHMED (T.), FAVRO (L. D.), THOMAS (R. L.) and HARTIKAINEN (J.) - Photoacoustic and Photothermal Phenomena, Springer Series in Optical Sciences, vol. 58, eds. Hess (P.) and Pelzl (J.) (Springer-Verlag, 1988), p. $415-$ 418.

[4] KUO (P. K.), AHMED (T.), FAVRO (L. D.), JIN (H. J.) and THOMAS (R. L.) - J. of Nondestructive Evaluation, vol. 8(2), 1989, p. 97-106.

[5] FAVRO (L. D.), AHMED (T.), JIN (H. J.), CHEN (P.), KUO (P. K.) and THOMAS (R. L.), Photoacoustic and Photothermal Phenomena, Springer Series in Optical Sciences, vol. 62, eds. Murphy (J. C.) et al. (Springer-Verlag, 1990), p. 490-492.

[6] WELCH (C. S.), and ZICKEL (M. J.) - Rev. of Progr. in Quantit. Nondestr. Eval., vol. 12, eds. Thompson (D. O.) and Chimenti (D. E.) (Plenum, NY, 1993), p. 1923-1930.

[7] ZICKEL (M. J.) and WELCH (C. S.), ibid, vol. 13, 1994, p. 1849-1855.

[8] BUSSE (G.), WU (D.), KARPEN (W.) -, J. Appl. Phys., vol. 71(8), 1992, p. 3962-3965.

[9] OFFERMANN (S.), MERIENNE (E.) and BEAUDOIN (J. L.) - NATO ASI Series; Series E, Applied Sciences, vol. 262, 1993, p. 43-48.

[10] OFFERMANN (S.), BISSIEUX (C.) and BEAUDOIN (J. L.) - Revue Générale de Thermique, vol. 37(8), 1998, p. 718-724.

[11] AGEMA INFRARED SYSTEMS, Insight, vol. 37(11), 1995, p. 860-861.

[12] POTET (P.) - CND par thermographie à démodulation synchrone à l'aide d'une caméra à matrice de détecteurs. Journée d'Etudes de la SFT, 22 January 1997. [in French]

[13] KRAPEZ (J.-C.), GARDETTE (G.) and BALAGEAS (D.) - Proc. 3rd Int. Workshop on Advanced IR Techn. and Appl., Capri (It.), Sept. 19-20, 1995, pub. Fondazione G. Ronchi (Firenze), 1995, p. 219-237.

[14] WU (D.), BUSSE (G.) - Revue Générale de Thermique, vol. 37(8), 1998, p. 693-703.

[15] FAVRO (L. D.), HAN (X.), KUO (P. K.), THOMAS (R. L.) - Proc. SPIE Thermosense XIX, Orlando FL, April 22-25, 1997, vol. 3056, p. 184-188. 
http://dx.doi.org/10.21611/qirt.1998.023
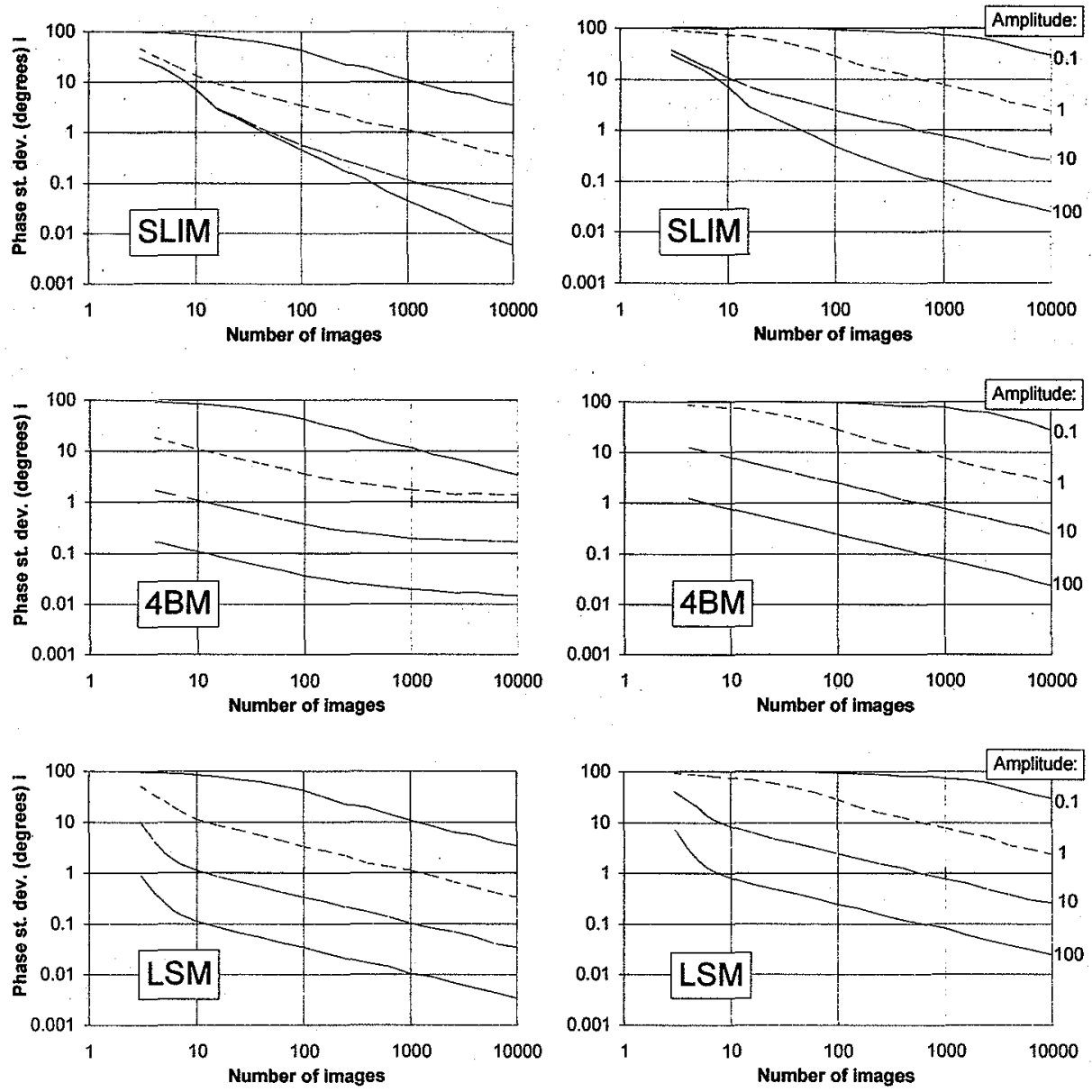

Fig. 1. Standard deviation of the phase lag measured by three lock-in methods for different values of the sinusoïdal signal amplitude (expressed in digit level) contaminated with either low level noise ( 0.3 digit - left) or medium level noise ( 3 digits - right) 
http://dx.doi.org/10.21611/qirt.1998.023
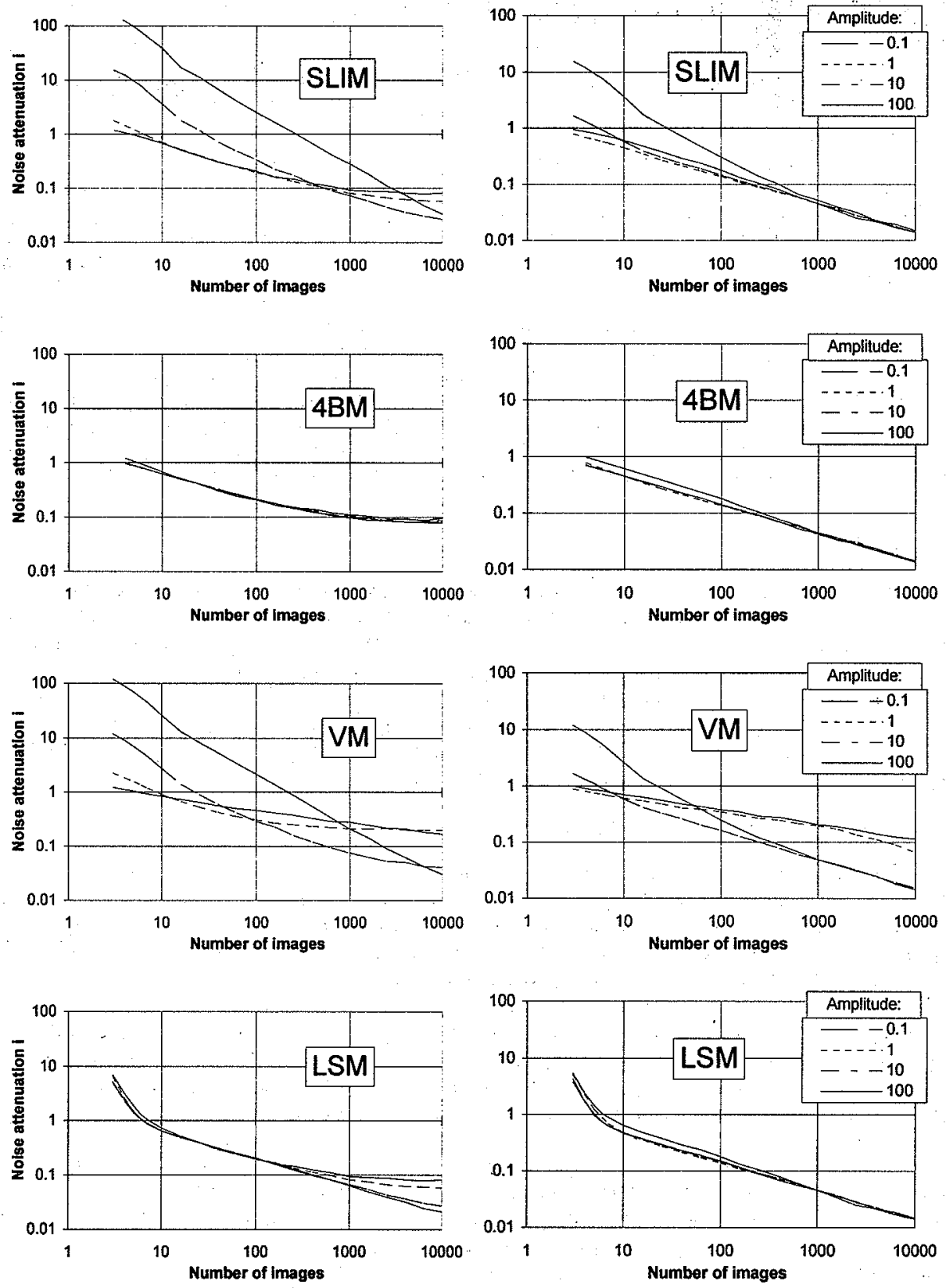

Fig. 2. Noise attenuation factor obtained by the four considered methods for different values of the sinusoïdal signal amplitude (expressed in digit level) contaminated with either low level noise ( 0.3 digit - left) or medium level noise ( 3 digits - right) 